\title{
Multiple paralogous genes related to the Streptomyces coelicolor developmental regulatory gene whiB are present in Streptomyces and other actinomycetes
}

\author{
J. A. Soliveri, ${ }^{1} \dagger$ J. Gomez, ${ }^{2} \neq$ W. R. Bishai ${ }^{2}$ and K. F. Chater ${ }^{1}$ \\ Author for correspondence: K. F. Chater. Tel: +44 1603 452571. Fax: +44 1603456844. \\ e-mail: chater@bbsrc.ac.uk
}

\footnotetext{
1 John Innes Centre, Norwich Research Park, Colney, Norwich NR4 7UH, UK

2 Dept of Molecular Microbiology and Centre for Tuberculosis Research, Johns Hopkins School of Hygiene and Public Health, $615 \mathrm{~N}$ Wolfe Street, Baltimore, MD 21205, USA
}

\begin{abstract}
The whiB sporulation gene of Streptomyces coelicolor was shown [Davis, N. K. \& Chater, K. F. (1992). Mol Gen Genet 232, 351-358] to encode a small, cysteinerich putative transcription factor unlike any that had been described previously. The large database of DNA sequences of mycobacteria (like Streptomyces, members of the Actinomycetales) has revealed a family of genes encoding proteins related to WhiB. Mycobacterium tuberculosis contains at least six such genes (whiB homologues in mycobacteria: whmA-F) and a likely seventh, whmG. Using conserved features of Whm proteins, a PCR-based approach led to the discovery that $S$. coelicolor A3(2) contains several similar genes. Cloning and sequencing of these whiB-like (wbl) genes revealed likely orthologues of four of the whm genes of M. tuberculosis. In all, S. coelicolor contains at least five wbl genes in addition to whiB itself. All five were shown by RT-PCR to be transcribed. A Southern blotting survey using each wbl gene as a probe showed that nearly all of a series of representatives of ten actinomycete genera (including morphologically simple organisms) contain close homologues of several wbl genes, suggesting that the ancient progenitor of all these organisms already contained a family of such genes, which have not been found in any other organisms.
\end{abstract}

Keywords: tuberculosis, leprosy, actinomycetes, prokaryotic phylogeny, sporulation in Streptomyces

\section{INTRODUCTION}

With the advent of 16S RNA sequencing, it has become clear that Gram-positive bacteria possessing high $\mathrm{G}+\mathrm{C}$ DNA form a distinct phylogenetic class, and the term Actinomycetes has been appropriated from its earlier more morphologically based usage to describe these organisms (Embley \& Stackebrandt, 1994). Two genera of actinomycetes, Mycobacterium and Streptomyces,

\footnotetext{
† Present address: Departamento de Microbiología y Parasitología, Facultad de Farmacía, Universidad de Alcalá, 28871-Alcalá de Henares, Spain.

$\ddagger$ Present address: Laboratory of Infection Biology, The Rockefeller University, Box 21, 1230 York Ave, New York, NY 10021, USA.

The GenBank accession numbers for the sequences reported in this paper are: wblA, AJ239085; wb/B, AJ239086; wblE, AJ239087; and wbll, AJ239088
}

have received particular attention: the genomes of Mycobacterium tuberculosis (Cole et al., 1998) and Mycobacterium leprae (Eiglmeier et al., 1993; Honoré et al., 1993; Fsihi et al., 1996; Smith et al., 1997), two of humankind's greatest scourges, have been completely or extensively sequenced, and the genome of Streptomyces coelicolor A3(2), a representative of the industrially important antibiotic-producing streptomycetes, is currently the subject of a genome sequencing project, to be completed during 2000 (www.sanger.ac.uk/Projects/ S_coelicolor/). This paper is concerned with the discovery of a family of genes in mycobacteria and streptomycetes that is also found in other actinomycetes, but is apparently absent from all other organisms studied.

Streptomycetes are developmentally complex (Chater \& Losick, 1997; Chater, 1998). They form mycelial colonies in which different physiological functions take 
place in different locations. Thus, most antibiotic synthesis takes place in the older parts of the substrate mycelium, whilst sporulation takes place in specialized aerial hyphae. Gene regulation and the possibility of development in mycobacteria are less well characterized, but there is some evidence that alterations in cell type may occur during infection by pathogenic mycobacteria (Chatterjee, 1976; Parrish et al., 1998).

The prototype of the gene family discussed in this paper, $w h i B$, is a developmental regulatory gene identified and characterized in $S$. coelicolor as being essential for sporulation of aerial hyphae (Davis \& Chater, 1992). It encodes an 87 amino acid polypeptide with attributes suggesting that it may be a DNA-binding protein. The sequence of WhiB contains four cysteines - a common motif in metal-coordinating DNA-binding proteins such as $\mathrm{Zn}$-binding GAL4 and other zinc finger proteins (Schjerling \& Holmberg, 1996) (including a class of small transcriptional activator proteins encoded by some phages; Julien et al., 1998), Fe-binding SoxR (Gaudu et al., 1997) and Hg-binding MerR (O’Halloran, 1993). Secondary structure analysis of WhiB indicated a potential helix-loop-helix structure from residues 64 to 84 (Davis \& Chater, 1992). The importance of this region was underscored by the fact that the whiB70 mutant, which contains a missense mutation leading to substitution of $\mathrm{Leu}_{74}$ by Pro, had the same phenotype as a whiB deletion mutant (Davis \& Chater, 1992). The whiB genes of Streptomyces aureofaciens (Kormanec et al., 1998) and 'Streptoverticillium griseocarneum' (Soliveri et al., 1993) function in a similar manner to whiB of S. coelicolor.

The existence of a mycobacterial gene encoding a WhiB homologue was first suggested by hybridization of a whiB-derived probe to restriction-endonucleasedigested Mycobacterium bovis BCG DNA (Soliveri et al., 1993). Subsequently, a whiB-like gene downstream of the groEL operon of M. tuberculosis was detected during database searches (Chater, 1993). The partial sequencing of the M. leprae genome (Honoré et al., 1993; Smith et al., 1997), and the recently completed sequencing of the M. tuberculosis genome (Cole et al., 1998) have provided an opportunity to scan large amounts of mycobacterial genomic DNA sequence for the presence of ORFs encoding homologues of WhiB. In this paper we describe multiple whiB homologues in $m y$ cobacteria (the whm gene family) and the use of this information to reveal their equivalents in Streptomyces (the $w b l$, for $w h i B$-like, gene family). Remarkably, this substantial gene family appears to be present in most (perhaps all) actinomycetes, yet absent from all other sufficiently characterized organisms.

\section{METHODS}

Strains, media and culture conditions. The Escherichia coli host strain used for routine subcloning was DH5 $\alpha$ (Hanahan, 1983). S. coelicolor M145, a plasmid-free prototrophic derivative of the wild-type strain A3(2) (Hopwood et al., 1985), was used as the source of DNA and of RNA for RT-PCR analysis. Media, conditions $\left(37^{\circ} \mathrm{C}\right.$, LB liquid medium, LBA solid medium) and media supplements (carbenicillin, $200 \mu \mathrm{g}$ $\mathrm{ml}^{-1}$; X-Gal, $40 \mu \mathrm{g} \mathrm{ml}^{-1}$ ) for E. coli were as described by Sambrook et al. (1989). Standard media and conditions for $S$. coelicolor were as described by Hopwood et al. (1985).

Genetic manipulation. For E. coli, plasmid preparation and transformation of competent cells were as described by Sambrook et al. (1989). For S. coelicolor, genomic DNA preparations were as described by Hopwood et al. (1985). Standard methods were used for restriction enzyme digestion, ligation, Southern blotting, PCR and radiolabelling of DNA (Sambrook et al., 1989) unless stated otherwise.

Screening of an S. coelicolor genomic library. An S. coelicolor genomic library of ordered cosmids (Redenbach et al., 1996) was screened by DNA hybridization using different genes as probes (Table 1). Probes of PCR products were amplified from the following plasmids: $w h m A_{M l}$, pTA577F [containing $w h m A_{M l}$, amplified by PCR from $M$. leprae cosmid B57, in pCRII (Invitrogen)] ; $w h m B_{M t}$, pTA63.3 (containing $w h m B_{M t}$, amplified by PCR from $M$. tuberculosis genomic DNA, in pCRII); $w h m C_{M l}$, pTA1937 (J. Gomez, unpublished); $w_{h m D_{M s}}$ pBS718 (J. Gomez, unpublished); whmE $E_{M t}$, pJS5001 $\left[w h m E_{M t}\right.$ was amplified by PCR from M. tuberculosis genomic DNA and inserted into the SmaI site of pIJ2926 (Janssen \& Bibb, 1993)]; saf (三wblI), pUL300 (Daza et al., 1990); whiB, pIJ558 (whiB gene in pUC19; Davis \& Chater, 1992). Probes were $\left[\alpha^{32} \mathrm{P}\right] \mathrm{dCTP}-$ labelled using an Oligolabelling Kit (Amersham Pharmacia Biotech), following the supplier's instructions. The cosmid library was probed under lowstringency conditions, prehybridization and hybridization being performed at $65^{\circ} \mathrm{C}$ in a solution containing $5 \times \mathrm{SSC}$, $5 \times$ Denhardt's solution (Hopwood et al., 1985), $0.5 \%$ (w/v) SDS and $100 \mu \mathrm{g}$ nonhomologous DNA ml ${ }^{-1}$. Hybridization overnight was followed by two washes in $2 \times$ SSC, $0 \cdot 1 \%$ SDS at $60^{\circ} \mathrm{C}$. After isolation, cosmid DNAs were further digested and a second Southern blot was performed.

Southern analysis of DNA of diverse actinomycetes for whmand whiB-like genes. Southern blots of restriction digests of mycobacterial DNA and a collection of diverse actinomycete chromosomal DNAs were hybridized with whm and wbl probes. Labelling, hybridization and detection were carried out at moderate stringency, using conditions described by Soliveri et al. (1993) except in the case of the studies shown in Fig. 1, when washing was in $3 \times \mathrm{SSC}, 0 \cdot 1 \%$ SDS at $55^{\circ} \mathrm{C}$ $(3 \times 20 \mathrm{~min})$. The probe to screen for the presence of whiB homologues in $S$. coelicolor was both generated and $[\alpha-$ $\left.{ }^{32} \mathrm{P}\right] \mathrm{dCTP}$-labelled by low-stringency PCR using genomic DNA from $S$. coelicolor as template and as primers, oligonucleotides WHB1 (5'-CTGGCAGGAGCGCGCACTGTG) and WHB2 (5'-TCGCTCGCATTCGGACAGGCC), which were designed on the basis of strongly conserved regions from the nucleotide and amino-acid sequence alignments of whiB and whm genes (the consensus conserved amino-acid sequences were, respectively, WQERALC and GLSERER). The primary PCR reaction mixture $(100 \mu \mathrm{l})$ contained $59 \cdot 5 \mu \mathrm{l}$ double-distilled $\mathrm{H}_{2} \mathrm{O}, 2 \mu \mathrm{l} 5 \mathrm{mM}$ dNTPs (A, G and T), $1 \mu \mathrm{l}$ $100 \mu \mathrm{M}$ dCTP, $5 \mu \mathrm{l}\left[\alpha^{3}{ }^{32} \mathrm{P}\right] \mathrm{dCTP}\left[3000 \mathrm{Ci} \mathrm{mmol}^{-1}(111 \mathrm{TBq}\right.$ $\left.\mathrm{mmol}^{-1}\right)$; Amersham], $1 \mu \mathrm{l}(20 \mu \mathrm{M})$ of each primer, $20 \mu \mathrm{l}$ $5 \times$ Buffer Q (Qiagen), $10 \mu \mathrm{l} 10 \times$ PCR Buffer (Qiagen) and $0.5 \mu \mathrm{l}(2.5 \mathrm{U}) \mathrm{Taq}$ Polymerase (Qiagen). In a Perkin-ElmerCetus DNA Thermal Cycler 9600, the amplification consisted of an initial $3 \mathrm{~min}$ denaturation step at $96^{\circ} \mathrm{C}$, and 32 cycles of $1 \mathrm{~min}$ at $96{ }^{\circ} \mathrm{C}, 1 \mathrm{~min}$ at $56^{\circ} \mathrm{C}$ and $2 \mathrm{~min}$ at $65^{\circ} \mathrm{C}$. The final extension step was $10 \mathrm{~min}$ at $65^{\circ} \mathrm{C}$. The wblG probe was generated by PCR amplification using the same DNA as 
Table 1. WhiB-like proteins of actinomycetes other than Streptomyces spp., identified through database searches

WhiB homologues were identified through searches of the non-redundant nucleotide and protein databases of the NCBI using the WhiB protein sequence as a query, and TBLASTN and the BLASTP option (gapped BLAST) of BLAST 2.0.8 (Altschul et al., 1997). Percentage identity indicates the number of matches observed per length of aligned sequences (i.e. residues at $\mathrm{N}$ and $\mathrm{C}$ termini of individual proteins extending beyond the other aligned protein are not included in this number).

\begin{tabular}{|llccc|}
\hline Protein & \multicolumn{1}{c}{ Source } & Length & $\begin{array}{c}\text { EMBL } \\
\text { accession } \\
\text { number }\end{array}$ & $\begin{array}{c}\text { Percentage } \\
\text { identity } \\
\text { to WhiB }\end{array}$ \\
\hline WhmA (whiB4, Rv3681c)* & Mycobacterium tuberculosis & $118 \neq$ & AL022121 & 37 \\
WhmA & Mycobacterium leprae & 119 & L01263 & 33 \\
WhmB (whiB3, Rv3416)* & Mycobacterium tuberculosis & 102 & Z77165 & 32 \\
WhmB & Mycobacterium leprae & 102 & $\mathrm{U} 00020$ & 33 \\
WhmC† & Mycobacterium tuberculosis & 96 & AL021646 & 22 \\
WhmC & Mycobacterium leprae & 89 & $\mathrm{U} 00016$ & 22 \\
WhmD (whiB2, Rv3260c)** & Mycobacterium tuberculosis & $123 \ddagger$ & AL021840 & 67 \\
WhmE (whiB1, Rv3219)** & Mycobacterium tuberculosis & 84 & Z95120 & 41 \\
WhmF (Rv3862c)* & Mycobacterium tuberculosis & $96 \neq$ & Z83864 & 25 \\
WhmG (Rv0022c)* & Mycobacterium tuberculosis & 139 & Z80233 & 22 \\
WblX (ORF6) & Rhodococcus opacus & 92 & AF030176 & 34 \\
\hline
\end{tabular}

*Designations of the corresponding genes given by Cole et al. (1998).

† This gene was not recognized as an ORF by Cole et al. (1998).

$\ddagger$ These lengths differ from those allocated in the EMBL database. See Fig. 5 legend for further details.

Table 2. Oligonucleotides used for RT-PCR amplifications of wb/ RNAs in S. coelicolor

\begin{tabular}{|llll|}
\hline Gene & \multicolumn{1}{c|}{ Sense oligonucleotide } & Antisense oligonucleotide & $\begin{array}{c}\text { Expected PCR } \\
\text { product (bp) }\end{array}$ \\
\hline$w b l A$ & 5'-GAA TAT GCC GCT GCC & 5'-CCC CTC GTT CGT ACT CCG TCC & 334 \\
$w b l B$ & 5'-CGT CCT TCG TCC ACT CCC C & 5'-GTG CCC AGT GGA TCG CAG & 309 \\
$w b l E$ & 5'-CGT TCA CAT TCA CAA GCA TCC & 5'-GTC CTC GCT GAG ACC ACC C & 249 \\
$w b l H$ & 5'-CGT TGA TAC CGC GTT GAG & 5'-GTT CCT TCT CCG ACA GGC & 268 \\
$w b l I$ & 5'-GGC GTT CCT GAG TTC CAC & 5'-TAG ACC CGT GCT GCA ACC & 348 \\
\hline
\end{tabular}

template and oligonucleotides WBG1 (5'-CGTTGATACCGCGTTGAG) and WBG2 (5'-GTTCCTTCTCCGACAGGC) as primers.

RT-PCR. Total RNA was isolated (Kelemen et al., 1996) from $S$. coelicolor exponential-phase cultures grown in YEME medium (Hopwood et al., 1985). To remove any residual DNA, RNA was treated with RNase-free DNase I (Boehringer Mannheim). The concentration of RNA was determined by $A_{260}$ measurements, and the quality of RNA was analysed on a conventional Tris/borate/ethidium bromide agarose gel. To control for the presence of contaminating DNA, samples containing $4 \mu \mathrm{g}$ of the RNA preparation, $20 \mathrm{pmol}$ of the same oligonucleotide and $1 \mu \mathrm{g}$ of RNase A (DNase-free; Boehringer Mannheim) were incubated, in a $20 \mu \mathrm{l}$ reaction volume, at $37^{\circ} \mathrm{C}$ for $1 \mathrm{~h}$. RT-PCR was performed using suitable oligonucleotides (Table 2) and a Titan One Tube RT-PCR Kit (Boehringer Mannheim) according to the supplier's instruc- tions (which include 10 rounds of reverse transcription and 25 rounds of PCR). Control samples containing genomic DNA instead of total RNA as the template were run as RT-PCR samples in parallel.

DNA sequencing and deduced protein analysis. The DNA sequence was determined with a Taq Dye Deoxy terminator cycle sequencing kit and a model 373A DNA sequencing system (Applied Biosystems). Nucleotide sequences and derived amino acid sequences were analysed with the GCG software package (version 8.1; Genetics Computer Group, Madison, WI, USA). Likely coding regions were revealed by the program Frame Plot, version 2.1 (www.nih.go.jp/ $\sim$ jun/cgi-bin/frameplot.pl). Protein secondary structure predictions were carried out using the Protein Sequence Analysis (PSA) server (bmerc-www.bu.edu/psa/) and the PredictProtein server, PHDsec (www.embl-heidelberg.de/ predictprotein/predictprotein.html\#P2SEC). 
Phylogenetic analysis of whiB-like genes. Amino acid sequences of the various $w h m$ and $w b l$ genes were aligned and analysed using the PROTDIST, FITCH and CONSENSE programs from the PHYLIP software package (Felsenstein, 1988). The dendrogram shown in Fig. 4 is the consensus tree of 500 runs of the FITCH program. Branch lengths are proportional to the genetic distance between proteins as assessed by PROTDIST.

Terminology (homologues, orthologues and paralogues). For the purpose of this paper, homologues (genes or their corresponding proteins that have an obvious common ancestry) can be broadly subdivided into orthologues (functionally equivalent homologues from different organisms) and paralogues (functionally related, but not equivalent, homologues arising from gene duplication within an evolutionary lineage) (Holland, 1999).

\section{RESULTS}

Database searches reveal a number of mycobacterial genes encoding WhiB-like proteins

Cole et al. (1998) identified four whiB-like genes in their annotation of the complete genome sequence of $M$. tuberculosis. We have identified a further three (Table 1). These seven whiB homologues of mycobacteria $(w \mathrm{hm}$ genes) would encode proteins ranging in identity to WhiB from 22 to $67 \%$ (Table 1). The four $M$. tuberculosis whm genes most closely related to whiB were described by Cole et al. (1998) as whiB1, whiB2, whiB3 and whiB4 (see Table 1), but we suggest that the use of the whm terminology will be less confusing and conform better to the conventions of bacterial genetics. The alphabetical order of the gene names $(w h m A-G)$ mainly reflects the order in which we became aware of their existence. Orthologues of $w h m A, B$ and $C$ are present in M. leprae (Honoré et al., 1993; Smith et al., 1997), and where necessary we distinguish between orthologues from different organisms by the use of subscripts. whmD is the most similar of the whm genes to $w h i B$. No orthologues of $w h m D$, whmE, whmF or whm $G$ have yet appeared in the publicly available $M$. leprae genome sequence, but the Mycobacterium smegmatis whmD gene has been cloned and characterized (J. Gomez \& W. R. Bishai, unpublished).

In most members of the Whm/Wbl family, a conserved set of cysteine residues is arranged as Cys-X(14-22)Cys-X2-Cys-X5-Cys (see below), but in WhmG ( $\equiv \mathrm{Rv} 0022 \mathrm{c}$ in the M. tuberculosis genome) the spacing of the corresponding residues is Cys-X11-Cys-X3-CysX7-Cys. Nonetheless, the other similarities to conserved regions of the $\mathrm{Whm} / \mathrm{Wbl}$ protein family are striking enough to warrant the designation of Rv0022c as whmG. Further sequence comparisons are made later in this paper.

\section{Multiple whm genes are also present in a rapidly growing, saprophytic mycobacterium, M. smegmatis}

M. smegmatis is saprophytic and has a significantly faster generation time than the pathogenic M. tuberculosis (3 vs 18-24 h in culture). Southern blotting experi-

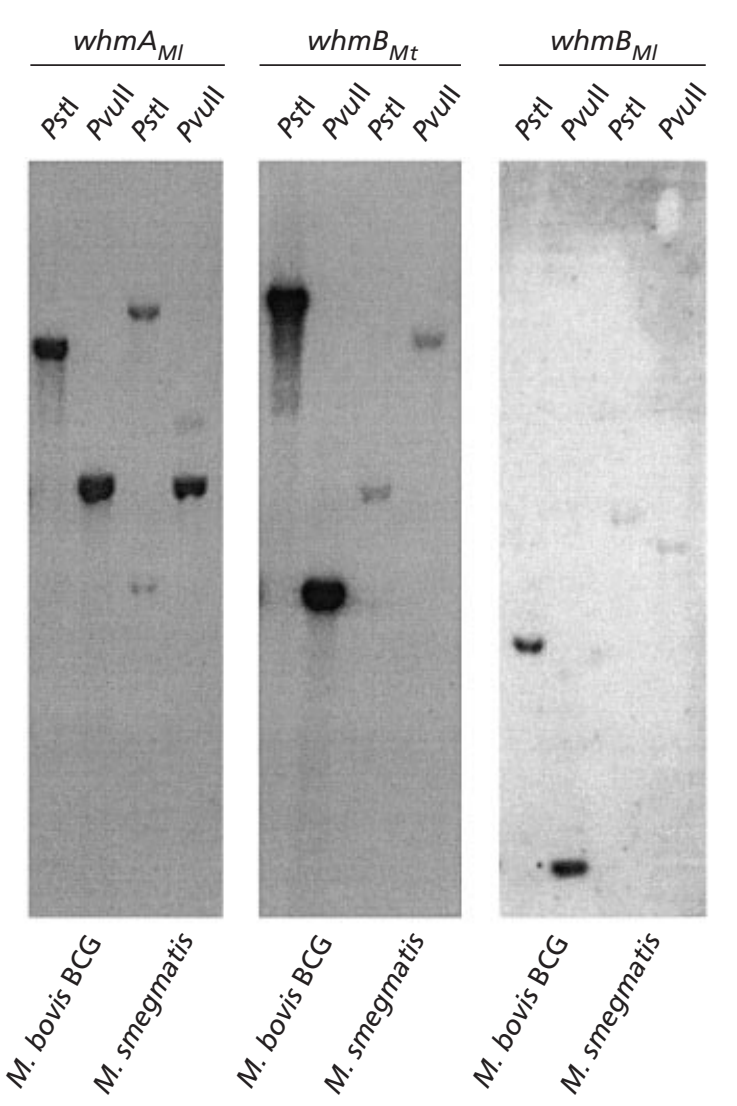

Fig. 1. Detection of $w h m$ homologues in $M$. smegmatis and $M$. bovis BCG. ${ }^{32} \mathrm{P}$-labelled probes to $w h m A_{M l}, w h m B_{M t}$ and whm $C_{M l}$ were generated by random priming of suitable restriction fragments. For further details see Methods.

ments on $M$. smegmatis DNA gave unique and strong signals with probes from $w h m A_{M l}$, $w h m B_{M t}$ and $w h m C_{M l}$ (Fig. 1), whmE (not shown) and whiB (revealing the previously cloned $w h m D_{M s}$; see above). Similar results (but different fragment sizes) were also obtained with DNA of M. bovis BCG (see Fig. 1).

\section{Multiple whiB-like genes are present in S. coelicolor}

The presence of paralogues of whiB in mycobacteria suggested that a similar situation might prevail in Streptomyces spp., and, in particular, in the extensively studied strain S. coelicolor A3(2). This would not only extend (and be informed by) the large amounts of genetic and biological information available for this strain, but might also in future help to evaluate the roles of whiB-like genes in mycobacteria. A firm indication that $S$. coelicolor has multiple whiB-like genes came from the use of a probe obtained by PCR using $S$. coelicolor DNA as template. The oligonucleotides designed as primers for PCR were based on two segments conserved among the known whiB-like genes (see Methods). Thus, it was hoped that several whiBlike genes would be represented in the PCR-amplified DNA. Indeed, the PCR-generated probe hybridized 

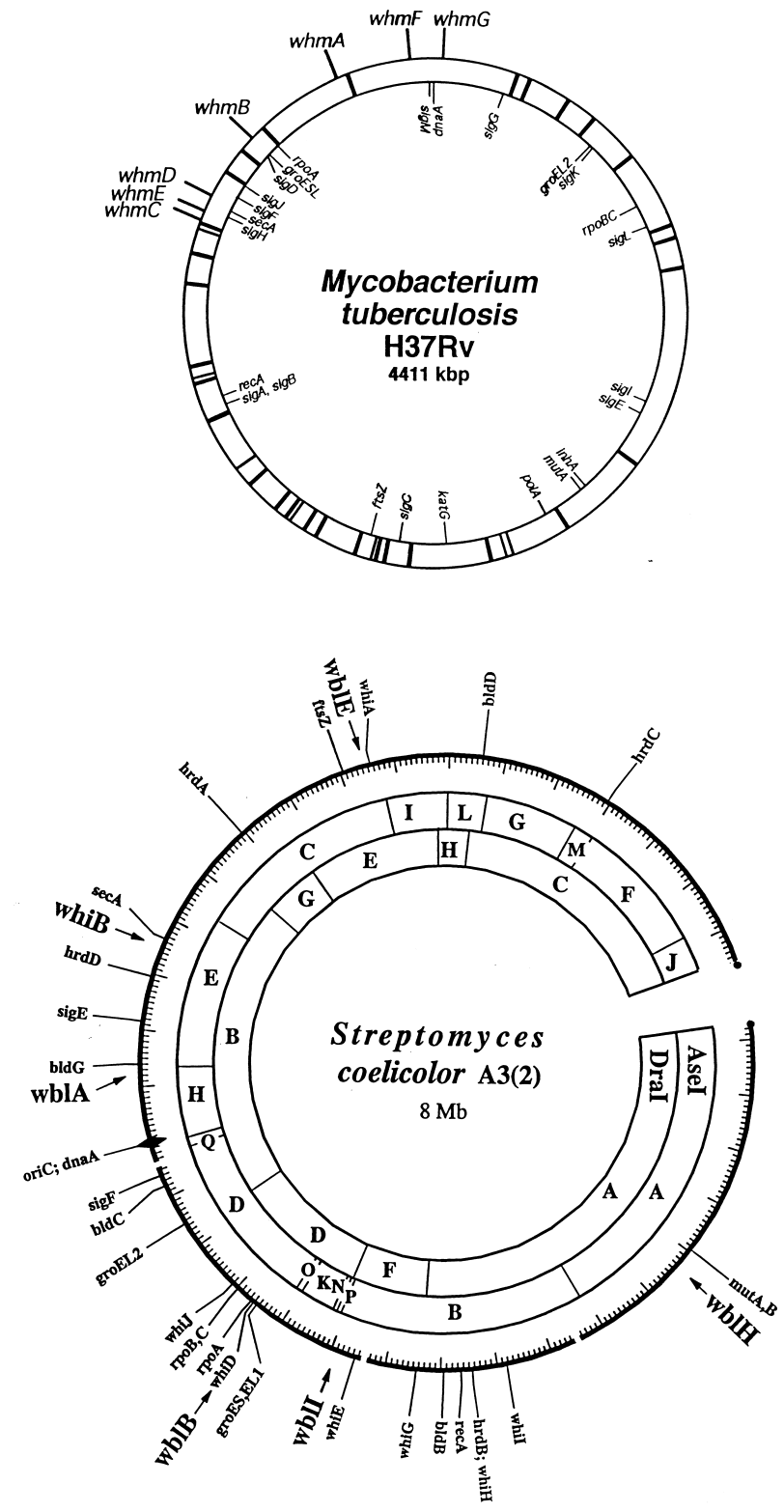

Fig. 2. Positions of $w h m$ and $w b /$ genes on the genomes of $M$. tuberculosis H37Rv and S. coelicolor A3(2). The H37Rv map is based on selected data from Cole et al. (1998) and is orientated

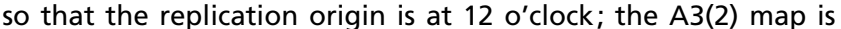
adapted from that of Redenbach et al. (1996) [the replication

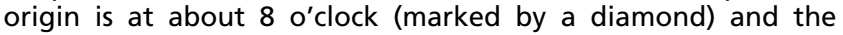
length of $8 \mathrm{Mb}$ is approximate because the genome is not yet fully sequenced]. The positions of $w b / A, w b / B$ and $w b / H$ have been confirmed by genomic sequence information from the Sanger Centre web site; that of whiB is based on genetic mapping (Chater, 1972); and those of wblE and wbll are provisional, based on their allocation to particular cosmids that have not yet been sequenced. The other genes shown were chosen on the slightly arbitrary basis that they encoded sigma factors or core RNA polymerase or had other particularly major roles in the cell or in morphological differentiation.

fairly strongly at six different positions to Southern blots of chromosomal DNA digests, one of which had the mobility expected for $w h i B$.
Thus encouraged, we used PCR products corresponding to each of five $w \mathrm{hm}$ genes ( $w \mathrm{hmA}$-whmE; see Methods) to detect $w \mathrm{hm}$ orthologues in S. coelicolor DNA. Each of these probes except the $w \mathrm{hm} C$ probe hybridized strongly to one band in Southern blots of S. coelicolor DNA digests, and each band corresponded to one of the bands obtained in the preliminary experiment. (Of the two other bands, one was later identified as wblH, which is described below, and the other remains unidentified.) The probes were then used to identify hybridizing cosmids in the $S$. coelicolor library, and after suitable subcloning we sequenced the relevant regions to reveal a $w h i B$-like $(w b l)$ gene in each case. With one exception (the $w h m D / w h i B$ pair) the genes have been given letters to match their likely equivalent whm genes. Thus, considering a conserved core segment corresponding to WhiB residues 24-80, WblA protein was $67 \%$ identical to WhmA; WblB was $66 \%$ identical to WhmB; no WhmC homologue was found (but see below); WhmD was $87 \%$ identical to WhiB; and WblE was $75 \%$ identical to WhmE. Furthermore, the ongoing $S$. coelicolor genome sequencing project (www.sanger.ac.uk/projects/S_coelicolor/) revealed another wbl gene in cosmid St4C6, which was not closely similar to any $w \mathrm{hm}$ genes and so was termed wblH (at the time of completing this paper, approximately $80 \%$ of the genome was available). $w b l H$ is identified as St4C6.25 (PID e1490484). The WblH protein is most similar to WhiB (58\% identity) and WhmD $(59.5 \%$ identity). In some cases, the corresponding $w h m$ and $w b l$ genes had similar genetic contexts. Thus, $w h m A_{M t}$ and $w b l A$ both diverge from genes encoding related putative penicillin-binding proteins; $w h m B_{M t}$ and $w b l B$ both converge on groEL, though $w b l B$ is separated from the convergent groEL1 operon by an intervening gene; and $w h m E_{M t}$ and $w b l E$ both converge on genes encoding related putative membrane sensor kinases. wblH, which has no close whm homologue, converges on a gene encoding a putative exported desaturase.

In summary, S. coelicolor contains close homologues (presumptive orthologues) of at least four of the seven known whm genes of mycobacteria. The seven whm genes of M. tuberculosis are noticeably clustered in less than $1 \mathrm{Mb}$ of the circular 4411529 base pair H37Rv chromosome, between positions $3468401(w \mathrm{hmC})$ and $27442(w h m G)$ (position 1, located near the origin of replication, is defined as the first base in the dnaA ORF); the homologues from $S$. coelicolor are more widely scattered and in a quite different order (Fig. 2), illustrating an absence of large-scale synteny between these diverged actinomycetes.

\section{Examination of the saf gene of Streptomyces griseus and its use as probe reveals another $w b /$ gene in S. coelicolor A3(2)}

Daza et al. (1990) identified a segment of S. griseus DNA (termed saf) that, when introduced into Streptomyces lividans on a multicopy plasmid, elicited pleiotropic 


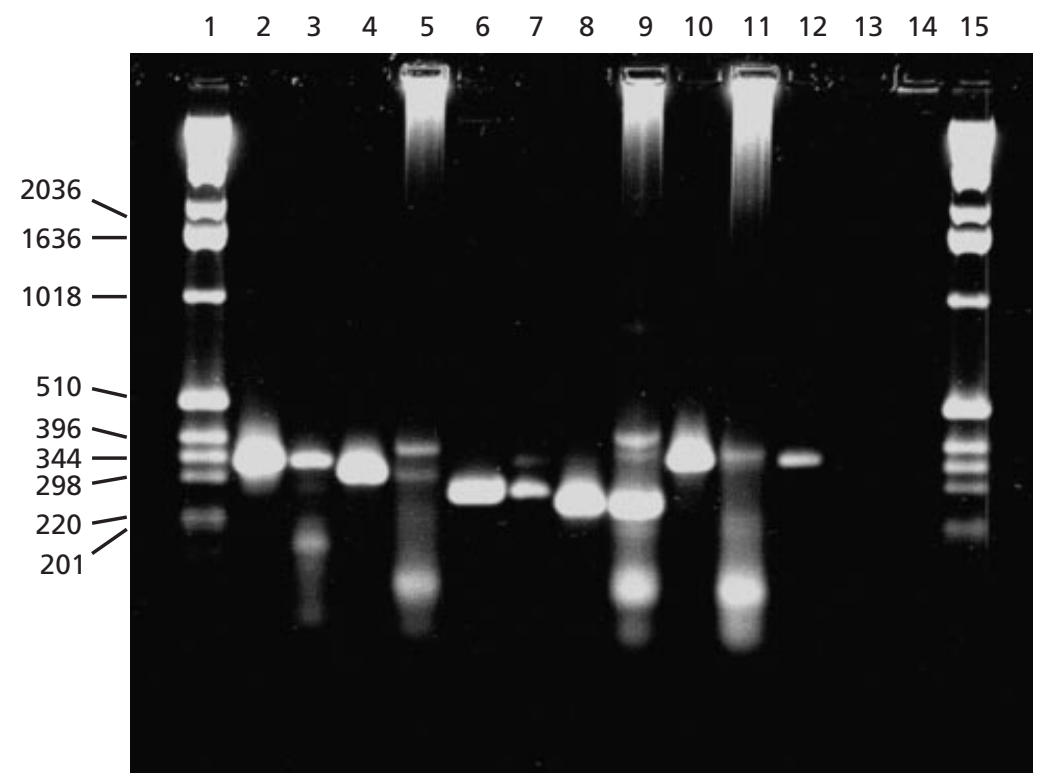

Fig. 3. Use of RT-PCR to demonstrate that all five $w b l$ genes of S. coelicolor M145 are transcribed. Lanes 1 and 15, DNA size standards (bp). Lanes 2, 4, 6, 8 and 10, RT-PCR of $w b / A, w b / B$, $w b / H$, $w b / E$ and $w b l l$, respectively, using genomic DNA as template. Lanes 3, 5, 7, 9 and 11, RT-PCR of $W b / A, w b / B, w b / H$, $W b / E$ and $w b l l$, respectively, using total RNA as template. Lanes 12, 13 and 14, controls: 12, wb/A RT-PCR of genomic DNA plus RNase, 13, wbIA RT-PCR of total RNA plus RNase, and 14, direct WbIA PCR amplification from total RNA without the use of reverse transcriptase. Details of primers are given in Table 2.

Table 3. Hybridization of $w b /$ probes to Southern blots of Pvull digests of DNA from representative actinomycetes

Strongly hybridizing bands are shown in bold, moderately strong bands in normal type and weak bands in square brackets.

\begin{tabular}{|c|c|c|c|c|c|c|}
\hline \multirow[t]{2}{*}{ Source of DNA in Southern blot } & \multicolumn{6}{|c|}{ Estimated size $(\mathrm{kb})$ of band hybridizing with different $\boldsymbol{w} \boldsymbol{b l}$ probes } \\
\hline & whiB & $\boldsymbol{w} b l A$ & $w b l B$ & $w b l E$ & $w b l H$ & $w b l I$ \\
\hline Micrococcus luteus & $1 \cdot 4$ & {$[5 \cdot 0]$} & {$[3 \cdot 8]$} & $4 \cdot 5$ & $1 \cdot 4$ & - \\
\hline Nocardioides simplex 2375 & $7 \cdot 0,5 \cdot 5$ & $4 \cdot 1,2 \cdot 8$ & - & $6 \cdot 0,4 \cdot 2,2 \cdot 9,2 \cdot 6$ & $8 \cdot 0,6 \cdot 2$ & - \\
\hline Actinoplanes brasiliensis & 13 & $0 \cdot 8$ & {$[1 \cdot 7]$} & $1 \cdot 4$ & {$[13]$} & - \\
\hline Micromonospora chalcea 2883 & $4 \cdot 8$ & 9 & $4 \cdot 2$ & {$[3 \cdot 1], 1 \cdot 8$} & $5 \cdot 8$ & - \\
\hline Rhodococcus fascians 2767 & $3 \cdot 2,2 \cdot 9$ & {$[12]$} & - & $9 \cdot 0,7 \cdot 0,4 \cdot 2$ & $3 \cdot 3,3 \cdot 1$ & - \\
\hline Mycobacterium bovis BCG & 3.9 & $1 \cdot 4$ & {$[1 \cdot 8]$} & {$[3 \cdot 0]$} & $4 \cdot 0,[3 \cdot 8]$ & - \\
\hline Mycobacterium smegmatis & {$[8 \cdot 0]$} & $3 \cdot 2$ & {$[7 \cdot 0]$} & {$[7 \cdot 2],[1 \cdot 3]$} & {$[10]$} & - \\
\hline Gordonia terrae & $5 \cdot 6$ & $9 \cdot 5$ & {$[5 \cdot 8],[1 \cdot 1]$} & $5 \cdot 2$ & $6 \cdot 2$ & - \\
\hline Saccharopolyspora erythraea & 15 & $5 \cdot 2$ & $2 \cdot 2$ & $3 \cdot 9$ & $?$ & - \\
\hline Amycolatopsis mediterranei 2747 & $?$ & - & $\mathbf{3} \cdot \mathbf{5},[2 \cdot 0]$ & - & - & - \\
\hline \multicolumn{7}{|l|}{ Streptomyces (various spp.) } \\
\hline Streptomyces coelicolor A3(2) (M145) & $1 \cdot 4$ & 5 & 10 & $5 \cdot 3$ & $5 \cdot 0,2 \cdot 4,1 \cdot 5$ & 5 \\
\hline Streptomyces antibioticus & $2 \cdot 8$ & 7 & $4 \cdot 0,0 \cdot 4$ & $3 \cdot 0,1 \cdot 7$ & 3 & $4 \cdot 8$ \\
\hline 'Streptoverticillium griseocarneum' & 1.6 & 5 & $0 \cdot 8$ & $2 \cdot 3$ & {$[1 \cdot 8]$} & $5 \cdot 3,1 \cdot 7$ \\
\hline Streptomyces griseus & $2 \cdot 9$ & $7 \cdot 2$ & $0 \cdot 5,0 \cdot 365$ & $1 \cdot 5$ & $8 \cdot 0,3 \cdot 2,2 \cdot 0$ & $4 \cdot 8$ \\
\hline Streptomyces albus $G$ & 3 & $3 \cdot 2$ & $1 \cdot 2$ & 5 & $2 \cdot 8$ & $4 \cdot 8$ \\
\hline
\end{tabular}

- , No signal.

?, Unclear.

changes in the production of exoenzymes and antibiotics. Surprisingly, routine database screening had revealed a whiB-like coding sequence extensively overlapping, but in the antisense strand of, the published saf coding sequence (Chater, 1993). Using a PCR-generated probe to this 'anti-saf' coding sequence, we cloned and then sequenced homologous DNA from the S. coelicolor cosmid library to reveal a gene that was designated $w_{b l I_{S c}}$. Both $w$ blI $_{S c}$ and the homologous 'anti-saf' sequence from $S$. griseus (which we term $w b l l_{S g}$ ) had potential ribosome-binding sites. Their deduced products were $78 \%$ identical end-to-end, and the DNA flanking wblI $_{S c}$ was closely similar to that in the $S$. griseus saf region, confirming that they were true orthologues. Frame plot analysis of the S. coelicolor sequence did not indicate that a saf-like gene was anti- 
sense to $w_{b l I_{S c}}$ : there was no suitable ribosome-binding site compared to that suggested for S. griseus, and a stop codon was present in the middle of what might have been the saf-like sequence of $S$. coelicolor. We therefore suggest that the wbll homologue of $S$. griseus may be responsible for the effects in S. lividans attributed to saf.

The discovery of $w$ bll brought the total number of $w h i B$ paralogues so far identified in $S$. coelicolor to six (including $w h i B$ itself).

\section{Evidence of transcription of $w b /$ genes}

To find out whether transcription occurred in $w b l$ genes during normal liquid culture (representing vegetative growth), a preparation of RNA isolated late in exponential growth of S. coelicolor A3(2) strain M145 was evaluated by RT-PCR, using primers specific to each $w b l$ gene (Table 2). In each case, a product of the predicted size was readily obtained, whilst control experiments showed no signal (Fig. 3). Thus, all of the $w b l$ genes are transcribed.

\section{Evidence that the $w b l$ gene family is present in many different actinomycetes}

The presence of apparent orthologues of most of the $w b l$ genes in mycobacteria and Streptomyces suggested that this gene family could be a general feature of actinomycetes. To test this, each $w b l$ gene (as well as whiB) was used as a probe against Southern blots of DNA from representatives of nine other actinomycete genera as well as four other Streptomyces spp. (Table 3). For nearly all DNA samples, at least one clearly hybridizing sequence was detected with most of the probes. Each sequence was located on a different restriction fragment, and each hybridized most strongly to one particular $w b l$ probe. Homologues of $w h i B$ and all five $w b l$ genes were present in all four streptomycetes. There were two main exceptions to the general presence of all the $w b l$ genes among actinomycetes: Amycolatopsis mediterranei hybridized strongly only with $w b l B$, and significant hybridization to wbll was confined to the five Streptomyces spp. tested. Undoubtedly, other $w b l$ genes remained undetected in this Southern blot analysis: for example, a gene (called here $w b l X_{R o}$ ) encoding a protein with $34 \%$ identity to whiB (and similarly distant from all known WhiB-like proteins) was discovered fortuitously in another actinomycete, Rhodococcus opacus, during the study of a quite different nearby gene (Seibert et al., 1998). The $w b l X_{R o}$ gene would not have been detected by any of the probes used in Table 3 .

\section{DISCUSSION}

\section{Implications of $w h m / w b /$ genes for theories of the evolution and phylogeny of actinomycetes}

Although conventional searches have revealed no whiBlike genes in any organisms other than actinomycetes, it is now clear that most actinomycetes contain several

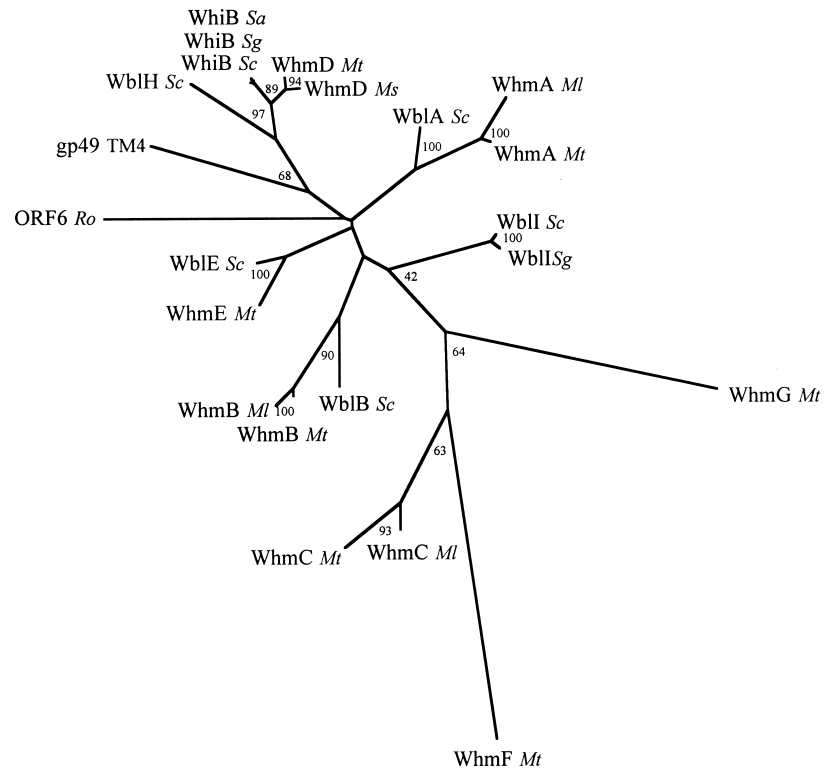

Fig. 4. Phylogeny of whiB-like proteins. The unrooted tree was constructed by using the SEQBOOT, PROTDIST, FITCH and CONSENSE programs from the PHYLIP software package. Nodes with bootstrap values $>40$ are indicated. Because only regions conserved among all WhiB-like proteins were included in the analysis, the Rhodococcus WbIX protein (here termed ORF6 Ro) does not cluster with any of the $\mathrm{Whm} / \mathrm{Wbl}$ proteins, despite the apparent similarities in length and sequence between residues 33-48 of WblX, the WhmB/WblB proteins and the Wbll proteins. The analysis includes the WhiB-like product of gene gp49 of the mycobacterial phage TM4 (Ford et al., 1998).

such genes. Molecular phylogenetic analysis (Fig. 4) of the genes discovered so far indicates that probable orthologues of at least four of the seven whm genes found in M. tuberculosis are present in Streptomyces. A limited (but wide-ranging) Southern blot analysis indicated that orthologues of at least four of the $w b l / w h m$ paralogues (probes $w b l A, w b l B, w b l E, w h i B$ ) are also present in most actinomycetes (Table 3). This makes it highly likely that the last ancestor common to all these genera, which is estimated to have existed nearly one billion years ago (Embley \& Stackebrandt, 1994), already possessed a set of these genes. Shared derived characters (synapomorphies) are the basis of phylogenetic theories, and $w b l$ genes can now join (at least) two other striking synapomorphies of actinomycetes: possession of a homologous approximately $100 \mathrm{nt}$ insertion in their 23S rRNA genes (Roller et al., 1992), and utilization of the carbohydrate-modified cysteine derivative mycothiol as their major antioxidant thiol, instead of the tripeptide glutathione (Newton et al., 1996). It is therefore important for the further understanding of early bacterial evolution to establish whether the $w b l$ or mycothiol synapomorphies are shared with any of the non-actinomycete descendants of even earlier evolutionary radiations such as Thermatoga, Thermus, Deinococcus, Thermomicrobium, Atopobium, Sphaerobacter and Bifidobacterium (the 


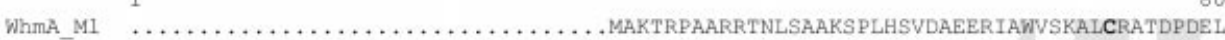

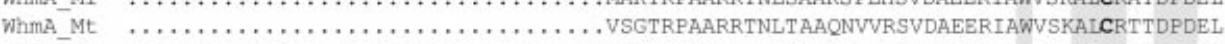

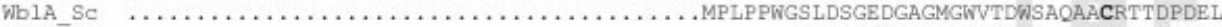

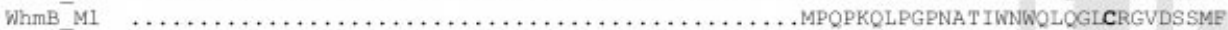

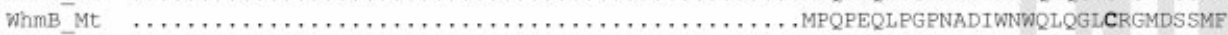

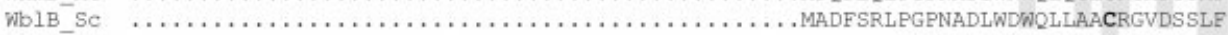

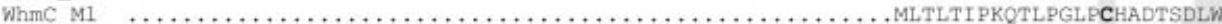

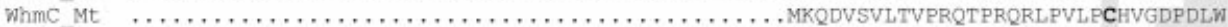

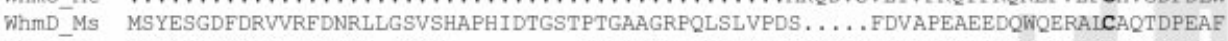
WhmD Mt MSYEHLRGVMGGTPHTTT . GSAT . . . . . . . ASATAVLRPHLSLVPEAPAPEEEPLPPEATDQWQDRA CAOTDPEAE

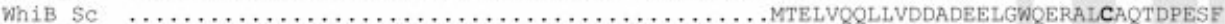

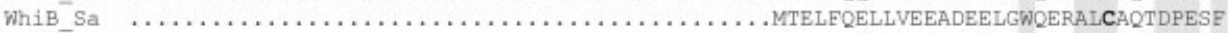

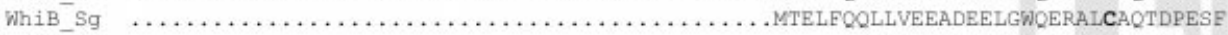

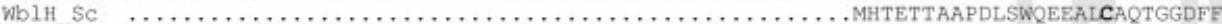

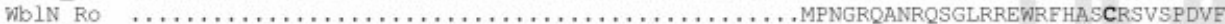

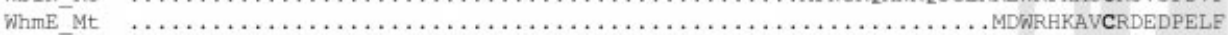

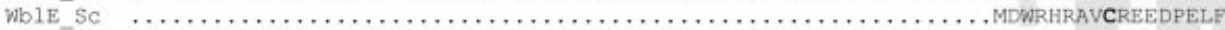

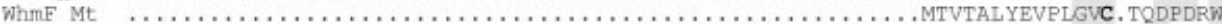

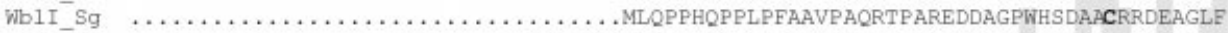

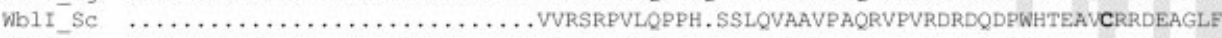

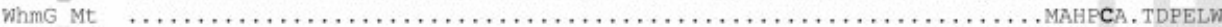
gp49_TM4

\section{Qd-helix}

$$
81
$$

WHB1

............RGAAORKAAVICR. HCPVM. . OECRADALDNKVEEGVWGGMTERQRGHCSSSIPRSCRGRISS EV .........RGAAORKAAVICR. HCPVM. OECAADALDNKVEFGVWGGMTERORRALLKOHPEVVSWSDYI FV $\ldots \ldots \ldots \ldots \ldots$ QGAAQNRAKAVCT , GCPVR. . TECLADALDNRVEFGVWGGMTERERRALLRRRPTVTSWRRLL EHPDG . . . . . . ERGRARMQREQRAKEMCR. RCPVI . EECRAHALDVGEPYGVWGGLSESERDLLLKGDLARSRSIPRS FHPDG . . . . . ERGRARTOREQRAKEMCR. RCPVI . EACRSHALEVGEPYGVWGGLSESERDLLLKGTMGRTRGIRRT FHPEG . . . . E. ERGAARSARENSAKEVCM, RCPVR . AECAAHALAVREPYGVWGGLTEDEREELMGRARNRLVAATAS FAETP ............... ADLECTKTLCA. NCPIR . RPCLEAAMERAEPWGVWGG. EIFDRGLIVSRKRPRGRPCNDV FADTP . . . . . . . AGLEVAKTLCV.SCPIR. . RQCLAAALQRAEPWGVWGG .EIEDQGSIVSHKRPRGRPRKDA FPEKG $\ldots \ldots \ldots \ldots \ldots$ GSTREAKRIC . GCEVR , DACLEYALAHDERFGIWGGLSERERRR. LKRGII $\ldots \ldots \ldots$ FPEKG $\ldots \ldots \ldots \ldots \ldots$ GSTREAKKICM. GCEVR . HECLEYALAHDEREGIWGGLSERERRR. LKRGII $\ldots \ldots \ldots$ FPEKG . . . . . . . . GSTREAKKVCL.ACEVR . SECLEYALANDEREGIWGGLSERERRR. LKKAAV . . . . . . FPEKG $\ldots \ldots \ldots \ldots \ldots$ GSTREAKKVCL. ACEVR . SECLEYGLANDEREGIWGGLSERERRR. LKKAAV $\ldots \ldots \ldots$ FPEKG $\ldots \ldots \ldots \ldots \ldots$ GSTREAKKVCL , ACEVR , SECLEYALANDEREG IWGGLSERERRR . LKKAAV $\ldots \ldots$ FPEPG. . . . . . . SSVRDAKRICA. ICPIR . NTCLEFALSNDEREGVWGGLSEKEPMA. LRRTIP . . . . . . FGPDG . . . . . ERYGPRIRREREAKRICS. SCLVL . ADCRAYAEESREGEGIWGGASEHERRA. LIGAVG . . . . . . FPVGN . . . . . . SGPALAQIADAKLVCN . RCPVT . TECLSWALNTGQDSGVWGGMSEDERRA . LKRRNARTKARTGV FPIGN ........ . TGPALLQIEEAKAVCR. RCPVM. .EQCLQWALESGQDSGVWGGLSEDERRA. MKRRAARNRARQAS TT . . . . . . W . W TPDDEAKTLCR. ACPRR. WLARDAVESAGAEGLWAGVVIPESGRARAFALGQLRSLAEF FAPSK . . . . . EPTAARLAREEAAKRVCA. RCPVM . VECQEHALMQPEPYGVWGGLTAAERRVALARRRRRDIELTAA FAPSK . . . . . . EPTAARLSREEA.AKRVCA . RCPVM . .VECREHALLQPEPYGVWGGLTAAERRVVLARRRRRDVELRNA FGYPDDDGSDGAAKARAYERSATQARIOCLRRCPLLQQRRCAOHAVEHRVEYGVWAGIKLPGGQYRKREQLAAAHDVLRR FPDKG $\ldots \ldots \ldots \ldots$, QSTRDAKRMCM, RCPLL , DECRALALRDPHLVGVWGGLSAQERRRIRKGASA $\ldots \ldots$

$\alpha$-helix $>$ L $\alpha$-helix LLL LLL $\alpha$-helix

161

WhmA_Ml

WhmA Mt

Wb1A_Sc

WhmB MI

WhmB_Mt

WblB_Sc

WhmC MI

Whmc_Mt

WhmD_Ms

WhmD Mt

WhiB Sc

WhiB Sa

WhiB Sg

WblH SC

Wb1N_Ro

WhmE_Mt

Wble SC

WhmF Mt

Wb1I_Sg

Wblisc

WhmG Mt

gP49_TM4

TRASTAMLAKFV $\ldots \ldots \ldots \ldots \ldots \ldots \ldots \ldots \ldots \ldots \ldots \ldots$

EKRKRRTGTAG . . . . . . . . . . . . . . . . .

ETARTEYERGVGIVPLDDDEVYENYAAVG $\ldots \ldots \ldots \ldots \ldots \ldots$

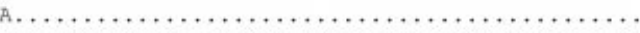

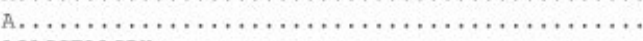

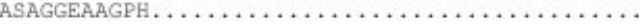

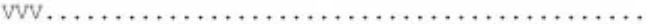

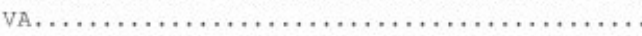

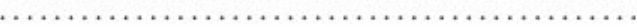

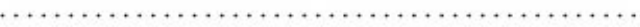

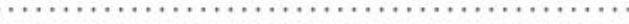

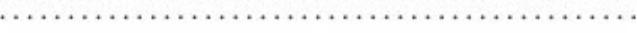

$\ldots \ldots+\ldots \ldots \ldots+\ldots+\ldots+\ldots, \ldots+\ldots, \ldots \ldots$

$\ldots, \ldots, \ldots, \ldots, \ldots, \ldots, \ldots, \ldots, \ldots, \ldots$,

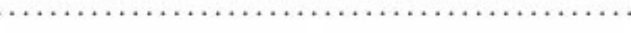

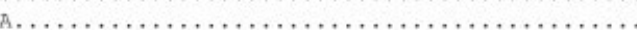

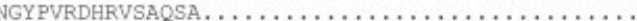

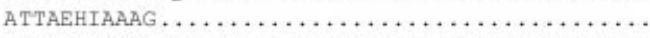

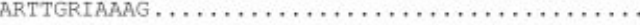

IAGGE INSRQLPDNAALLARNEGLEVT PVPGVVVHLPIAQVGPQPAA

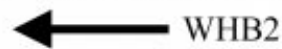

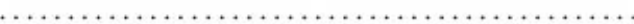

Fig. 5. Alignment of known WhiB-like proteins and their predicted structural features. Shown are the deduced amino acid sequences of 3 WhiB proteins and 19 other WhiB-like proteins. In three cases, the choice of the N-terminal methionine residue of $\mathrm{Whm}_{M t}$ proteins differs from that suggested in the EMBL annotation of the M. tuberculosis 
order of this list reflects increasing closeness to actinomycetes; Embley \& Stackebrandt, 1994). Notably, the wbll family seems to be confined to Streptomyces spp. Possibly, this may indicate that it fulfils a role peculiar to Streptomyces.

It is interesting to note the presence of a whiB-like gene, gp49, in a recently sequenced mycobacterial phage genome (Tm4; Ford et al., 1998: Figs 4 and 5), which raises the possibility that comparatively recent phagemediated horizontal transfer of whiB-like genes might have occurred; however, this could not readily account for the presence of similar sets of several such genes in nearly all actinomycetes. Small transcriptional activator proteins containing four cysteine residues, albeit with quite different spacing (Cys-X2-Cys-X23-Cys-X4-Cys) from the array of conserved cysteines in the WhiB family (and showing no other similarities to WhiB) are specified by several phages of Gram-negative bacteria (Julien et al., 1998).

\section{Structure-function predictions for WhiB-like proteins}

The WhiB-like proteins are aligned in Fig. 5. They are all small (from 87 to 130 amino acid residues). They all possess a high overall hydrophilicity suggestive of a cytoplasmic location. Although they all possess an overall negative charge, they have positively charged regions near their carboxy-termini. All the proteins are predicted to contain extensive $\alpha$-helical structure with a central $\beta$-sheet region between the first and second $\alpha$ helices. The predicted $\alpha$-helical regions all show periodic conservation of residues consistent with a face-of-thehelix arrangement, such that the conserved features (mostly hydrophobic and including the four conserved cysteines) are on one face, and the non-conserved (more often hydrophilic) are on the other (the changed spacing of cysteine residues in $\mathrm{WhmG}_{M t}$ would still permit this arrangement). Such arrangements suggest that the conserved hydrophobic faces may be involved in interactions within the protein, and the more variable hydrophilic faces may make contact with other molecules in the surrounding cytoplasm. The most Cterminal $\alpha$-helix, which always contains a segment rich in basic residues, is a candidate for DNA-binding (Suzuki, 1993), but probably with different targets among the different members of the family, since this $\alpha$ helical region is not very highly conserved in primary sequence between paralogues (in contrast to its much greater conservation between $\mathrm{Whm}$ and $\mathrm{Wbl}$ orthologues). A short loop region between the two most Cterminal ' $\alpha$-helical' regions contains a few residues closely similar within orthologues but differing among paralogues, followed by a universally conserved turn region (GV/IWGG). This loop between $\alpha$-helical regions is likely to be exposed, and the conserved turn region is a prime candidate for an interaction with another conserved cellular component (perhaps RNA polymerase, bound adjacent to a $\mathrm{Wbl}$ protein at a promoter).

The presence of four conserved cysteine residues suggests that the $\mathrm{Whm} / \mathrm{Wbl}$ proteins may be sensitive to redox changes, perhaps through a bound metal atom or through direct sensitivity to oxidation via disulphide bond formation, as in the OxyR transcription factor of E. coli (Zheng et al., 1998). To date, these possibilities have been difficult to address experimentally because no biochemical activity or cellular target for the activity of any of the proteins has been defined. Nevertheless, the possession of these genes by actinomycetes, and the reliance of actinomycetes on mycothiol in place of glutathione, may be no coincidence; both may perhaps reflect a specialization coinciding with biologically very significant increases in oxygen concentration believed to have occurred in the earth's atmosphere around 700-800 million years ago, and to have permitted the emergence of fully aerobic bacteria (Ochman \& Wilson, 1987).

In summary, we speculate that the proteins are transcription factors that activate different target genes, possibly sensing redox changes that could originate from environmental change or be generated internally during metabolic shift-down (for example, at the end of vegetative growth or when aerial hyphae switch from growth to sporulation) (Nyström, 1999).

Studies of the effects of $w b l$ mutations in the genetically manipulable $S$. coelicolor A3(2) may help to illuminate the functions of $w \mathrm{hm}$ genes in pathogenic mycobacteria. A particular hope is that the epidemiologically important, but little understood phenomenon of mycobacterial dormancy within hosts may share regulatory features with the extensively studied process of sporulation in Streptomyces, in which whiB plays a very important part. Intriguingly, it has been demonstrated that $M$. tuberculosis is capable of achieving a dormant state in vitro following a gradual shift to anaerobic conditions (Lim et al., 1999). The presence of $w \mathrm{hm}$ genes in $M$. smegmatis, a non-pathogen, suggests that the whm genes do not play a specific role in mycobacterial virulence, although indirect or contributory roles in virulence cannot be ruled out. At least one whm gene $\left(w h m D_{M s}\right)$ is essential for colony formation by $M$. smegmatis (J. Gomez \& W. R. Bishai, unpublished), whereas a very recent paper (Hutter \& Dick, 1999)

genome sequence (accession numbers AL022121, AL123456) because of the placement of potential ribosome-binding sites: $\mathrm{WhmA}_{M t}$ has gained 20 residues, which align well with the $\mathrm{N}$-terminus of $\mathrm{WhmA}_{M l} ; \mathrm{WhmD}_{M t}$ has gained 34 residues, which align fairly well with the $\mathrm{N}$-terminus of $\mathrm{WhmD}_{M s}$; and $\mathrm{WhmF}_{M t}$ has lost 20 residues. Shading indicates residues similar in $>50 \%$ of the proteins. Arrows indicate the regions used to design oligonucleotides for PCR amplification of whiB-like genes and the limits of the region used for the phylogenetic analysis in Fig. 4. MI, Mycobacterium leprae; Mt, Mycobacterium tuberculosis; Ms, Mycobacterium smegmatis; Ro, Rhodococcus opacus; Sa, Streptomyces aureofaciens; Sc, Streptomyces coelicolor; Sg, 'Streptoverticillium griseocarneum'; TM4, mycobacterial phage TM4; L, predicted loop region. 
reported that a $w h m B$ homologue of M. smegmatis did not affect growth or dormancy.

\section{NOTE ADDED IN PROOF}

Mulder et al. (1999) provide evidence that $w h m B_{M t}$ ( $\equiv$ whiB3) is expressed throughout growth, but expression from each of three promoters is growthphase-dependent.

\section{ACKNOWLEDGEMENTS}

We thank J. A. Gil (Universidad de León, Spain) for plasmid pUL300 and helpful information about the saf gene; Tobias Kieser for help with preparing some of the figures; and José Aínsa, Mark Buttner, David Hopwood, Gabriella Kelemen, Tobias Kieser and Sebastien Mouz for comments on the manuscript. This work was supported in part by CICYT (BIO95-1558-C02-02), Spain. J. Soliveri was supported by Ministerio de Educación y Ciencia, 'Estancias de Investigadores Españoles en Centros de Investigación Extranjeros (PR95-382)', Spain. Work at Johns Hopkins University was supported by NIH grants AI 36973 and AI 37856. Work at the John Innes Centre was funded by a competitive strategic grant from the Biotechnological and Biological Sciences Research Council and by the John Innes Foundation.

\section{REFERENCES}

Altschul, S. F., Madden, T. L., Schaffer, A. A., Zhang, J., Zhang, Z., Miller, W. \& Lipman, D. J. (1997). Gapped BLAST and PSI-BLAST: a new generation of protein database search programs. Nucleic Acids Res 25, 3389-3402.

Chater, K. F. (1972). A morphological and genetic mapping study of white colony mutants of Streptomyces coelicolor. J Gen Microbiol 72, 9-28.

Chater, K. F. (1993). Genetics of differentiation in Streptomyces. Annu Rev Microbiol 47, 685-713.

Chater, K. F. (1998). Taking a genetic scalpel to the Streptomyces colony. Microbiology 144, 1465-1478.

Chater, K. F. \& Losick, R. (1997). The mycelial life-style of Streptomyces coelicolor A3(2) and its relatives. In Bacteria as Multicellular Organisms, pp. 149-182. Edited by J. H. Shapiro \& M. Dworkin. New York: Oxford University Press.

Chatterjee, B. R. (1976). Carrier state in leprosy. Lepr India 48, 643-644.

Cole, S. T., Brosch, R., Parkhill, J. \& 39 other authors (1998). Deciphering the biology of Mycobacterium tuberculosis from the complete genome sequence. Nature 393, 537-544.

Davis, N. K. \& Chater, K. F. (1992). The Streptomyces coelicolor whiB gene encodes a small transcription factor-like protein dispensable for growth but essential for sporulation. Mol Gen Genet 232, 351-358.

Daza, A., Gil, J. A., Vigal, T. \& Martin, J. F. (1990). Cloning and characterization of a gene of Streptomyces griseus that increases production of extracellular enzymes in several species of Streptomyces. Mol Gen Genet 222, 384-392.

Eiglmeier, K., Honoré, N., Woods, S. A., Caudron, B. \& Cole, S. T. (1993). Use of an ordered cosmid library to deduce the genomic organization of Mycobacterium leprae. Mol Microbiol 7, 197-206.
Embley, T. M. \& Stackebrandt, E. (1994). The molecular phylogeny and systematics of the actinomycetes. Annu Rev Microbiol 48, 257-289.

Felsenstein, J. (1988). Phylogenies from molecular sequences: inference and reliability. Annu Rev Genet 22, 521-565.

Ford, M. E., Stenstrom, C., Hendrix, R. W. \& Hatfull, G. F. (1998). Mycobacteriophage TM4: genome structure and gene expression. Tuber Lung Dis 79, 63-73.

Fsihi, H., De Rossi, E., Salazar, L. \& 7 other authors (1996). Gene arrangement and organization in a $\sim 76 \mathrm{~kb}$ fragment encompassing the oriC region of the chromosome of Mycobacterium leprae. Microbiology 142, 3147-3161.

Gaudu, P., Moon, N. \& Weiss, B. (1997). Regulation of the soxRS oxidative stress region: reversible oxidation of the $\mathrm{Fe}-\mathrm{S}$ centres of SoxR. J Biol Chem 272, 5082-5086.

Hanahan, D. (1983). Studies on transformation of Escherichia coli with plasmids. J Mol Biol 166, 557-580.

Holland, P. W. (1999). The effect of gene duplication on homology. Novartis Found Symp 222, 226-236.

Honoré, N., Bergh, S., Chanteau, S. \& 7 other authors (1993). Nucleotide sequence of the first cosmid from the Mycobacterium leprae genome project: structure and function of the Rif-Str regions. Mol Microbiol 7, 207-214.

Hopwood, D. A., Bibb, M. J., Chater, K. F. \& 7 other authors (1985). Genetic Manipulation of Streptomyces: a Laboratory Manual. Norwich: John Innes Foundation.

Hutter, B. \& Dick, T. (1999). Molecular genetic characterisation of whiB3, a mycobacterial homologue of a Streptomyces sporulation factor. Res Microbiol 150, 295-301.

Janssen, G. R. \& Bibb, M. J. (1993). Derivatives of pUC18 that have $B g l I I$ sites flanking a modified multiple cloning site and that retain the ability to identify recombinant clones by visual screening of Escherichia coli colonies. Gene 124, 133-134.

Julien, B., Pountney, D., Christie, G. E. \& Calendar, R. (1998). Mutational analysis of a satellite phage activator. Gene 223, 129-134.

Kelemen, G. H., Brown, G. L., Kormanec, J., Potuckova, L., Chater, K. F. \& Buttner, M. J. (1996). The positions of the sigma factor genes, whiG and sigF, in the hierarchy controlling the development of spore chains in the aerial hyphae of Streptomyces coelicolor A3(2). Mol Microbiol 21, 593-603.

Kormanec, J., Sevcikova, B., Sprusansky, O., Benada, O., Kofronova, O., Novakova, R., Rezuchova, B., Potuckova, L. \& Homerova, D. (1998). The Streptomyces aureofaciens homologue of the $w h i B$ gene is essential for sporulation; its expression correlates with the developmental stage. Folia Microbiol 43, 605-612.

Lim, A., Eleuterio, M., Hutter, B., Murugasu-Oei, B. \& Dick, T. (1999). Oxygen depletion-induced dormancy in Mycobacterium bovis BCG. J Bacteriol 181, 2252-2256.

Mulder, N. J., Zappe, H. \& Steyn, L. M. (1999). Characterization of a Mycobacterium tuberculosis homologue of the Streptomyces coelicolor whiB gene. Tuber Lung Dis 78, 299-308.

Newton, G. L., Arnold, K., Price, M. S. \& 7 other authors (1996). Distribution of thiols in microorganisms: mycothiol is a major thiol in most actinomycetes. J Bacteriol 178, 1990-1995.

Nyström, T. (1999). Starvation, cessation of growth and bacterial aging. Curr Opin Microbiol 2, 214-219.

Ochman, H. \& Wilson, A. C. (1987). Evolutionary history of enteric bacteria. In Escherichia coli and Salmonella typhimurium: Cellular and Molecular Biology, pp. 1649-1654. Edited by F. C 
Neidhardt and others. Washington, DC: American Society for Microbiology.

O'Halloran, T. V. (1993). Transition metals in control of gene expression. Science 261, 715-725.

Parrish, N., Dick, J. \& Bishai, W. R. (1998). Mechanisms of latency in Mycobacterium tuberculosis. Trends Microbiol 6, 107-112.

Redenbach, M., Kieser, H. M., Denapaite, D., Eichner, A., Cullum, J., Kinashi, H. \& Hopwood, D. A. (1996). A set of ordered cosmids and a detailed genetic and physical map for the $8 \mathrm{Mb}$ Streptomyces coelicolor A3(2) chromosome. Mol Microbiol 21, 77-96.

Roller, C., Ludwig, W. \& Schleifer, K. H. (1992). Gram-positive bacteria with a high DNA G $+\mathrm{C}$ content are characterized by a common insertion within their $23 \mathrm{~S}$ rRNA genes. J Gen Microbiol 138, 1167-1175.

Sambrook, J., Fritsch, E. F. \& Maniatis, T. (1989). Molecular Cloning: a Laboratory Manual, 2nd edn. Cold Spring Harbor, NY: Cold Spring Harbor Laboratory.

Schjerling, P. \& Holmberg, S. (1996). Comparative amino acid sequence analysis of the C6 zinc cluster family of transcription regulators. Nucleic Acids Res 24, 4599-4607.
Seibert, V., Kourbatova, E. M., Golovleva, L. A. \& Schlomann, M. (1998). Characterization of the maleylacetate reductase MacA of Rhodococcus opacus 1CP and evidence for the presence of an isofunctional enzyme. J Bacteriol 180, 3503-3508.

Smith, D. R., Richterich, P., Rubenfield, M. \& 22 other authors (1997). Multiplex sequencing of $1.5 \mathrm{Mb}$ of the Mycobacterium leprae genome. Genome Res 7, 802-819.

Soliveri, J., Granozzi, C., Plaskitt, K. A. \& Chater, K. F. (1993). Functional and evolutionary implications of a survey of various actinomycetes for homologues of two Streptomyces coelicolor sporulation genes. J Gen Microbiol 139, 2569-2578.

Suzuki, M. (1993). Common features in DNA recognition helices of eukaryotic transcription factors. EMBO J 12, 3221-3226.

Zheng, M., Åslund, F. \& Storz, G. (1998). Activation of the OxyR transcription factor by reversible disulfide bond formation. Science 279, 1718-1721.

Received 21 July 1999; revised 15 October 1999; accepted 27 October 1999. 